La perte d'humidité en 12 jours a été ainsi de $16 \%$.

La perte au moulage, par suite du mauvais caillé obtenu a été très forte. En effet, la matière sèche totale des $11 \mathrm{~kg}$. de poudre employée $92,21 \times 11$

étant de $\frac{92,21 \times 11}{100}=10 \mathrm{~kg}, 143$ et le poids total de la matière sèche 100

contenue dans les 78 fromages étant de $\frac{7.750 \times 38}{100}=2 \mathrm{~kg} .945$,

il y a donc eu perte de $10.143-2,945=7 \mathrm{~kg}$. 198 de matière sèche.

Cette perte provient du fait que le caillé, étant trop mou, s'est échappé par les trous des moules, entrainé par le petit-lait d'égouttage.

Cet essai, malgré la perte formidable de caillé qui s'est produite malgré tout très intéressant même, au point de vue économique.

En effet, si nous comptons le lait en poudre écrémé à raison de $4 \mathrm{fr}$. le $\mathrm{kg}$ et le prix de vente des Saint-Marcellin à $8 \mathrm{fr}$. le $\mathrm{kg}$. nous obtenons en adoptant les chiffres de cet essai :

$$
\begin{gathered}
\text { Valeur de } 11 \mathrm{~kg} \text {. de poudre : } 4 \times 11= \\
\text { Valeur de } 7 \mathrm{~kg} \text {. } 750 \text { de fromages à } 8 \mathrm{fr} . \\
\text { Bénéfice brut }
\end{gathered}
$$

La seule difficulté réside actuellement dans la coagulation du lait reconstitué. Le jour où sa coagulation méthodique sera résolue, les plus grands espoirs seront permis et la portée économique de cette nouvelle industrie incalculable.

$$
*^{*} *
$$

Ces essais seront continués avec des poudres plus fraîches, bien conditionnées et qui n'auront pas trainé dans des pièces plus ou moins humides, comme l'avait fait le lait sec qui a servi aux essais ci-dessus.

Nous serons heureux de tenir les lecteurs de la Revue Le Lait au courant de nos essais ultérieurs.

\title{
LES EAUX RÉSIDUAIRES DES LAITERIES
}

\author{
par Paul ROSSI, \\ Directeur des Services Vétérinaires de Saône-et-Lolre
}

L'extension rapide et imprévue de l'industrie laitière a fait apparaître, dans toutes les régions de France, un lourd problème dont la solution, encore incomplète, ne pourra devenir définitive qu'après de nouvelles et patientes recherches; c'est celui de l'évacuation des eaux résiduaires.

Ce problème très complexe, dont les conséquences pour l'hygiène sont graves, n'a attiré l'attention des hygiénistes qu'à la suite de sérieux 
incidents : pollution des rivières, pollution des sources, pullulation des moustiques, etc... Il préoccupe aussi bien les grands dépôts de lait et les fromageries que les beurreries-caséineries. Dans ce court exposé, nous l'envisagerons plus spécialement du point de vue de ces dernières, dont nous avons pu apprécier et étudier l'organisation pendant notre séjour en Charente-Inférieure, berceau des laiteries coopératives. C'est en effet à Chaillé, près de Surgères, qu'en 1888 , le génial BIRAUD fondait la première laiterie, rendant ainsi la prospérité à une région ruinée par le phylloxéra. Depuis cette date, ces établissements, parfaitement outillés pour la fabrication du beurre et l'extraction de la caséine, se sont multipliés et dépassent maintenant le nombre de 54 pour ce seul département charentais.

Tout d'abord, qu'entend-on par eaux résiduaires de laiterie? Ce sont :

$1^{0}$ L'eau des chaudières ;

$2^{\circ}$ L'eau des machines à glace;

$3^{\circ}$ Les eaux de laiterie, proprement dites.

L'eau des chaudières est stérile; une ébullition prolongée l'a, aussi, débarrassée de son tartre; c'est pour cette raison qu'elle est récupérée et sert à nouveau. Nous la passerons sous silence.

L'eau des machines à glace ne peut avoir le moindre contact avec le lait ou ses sous-produits ; elle est done pure et peut être déversée, sans danger, directement dans une rivière, un canal, un fossé ou mieux être récupérée.

Les eaux de laiterie, proprement dites, proviennent du lavage et du rinçage des bidons et récipients, ayant servi au transport du lait; du lavage des appareils de fabrication du beurre et enfin du lavage de la caséine. Souvent même, dans certaines régions où, à tort, la valeur du petit-lait et du babeurre pour l'alimentation et l'engraissement intensif des pores est encore négligée, ces sous-produits viennent augmenter à la fois la masse des eaux résiduaires et leur richesse en matières organiques. Dans l'Ouest et en particulier en Charente-Inférieure, l'industrie porchère les utilise à peu près intégralement ; il n'y sont jamais mélangés aux eaux résiduaires.

Toutes, riches en matières organiques, les eaux de laiterie ont une composition très variable, qui dépend essentiellement de leur provenance et du genre d'opérations auxquelles se livrent les laiteries. Leur teneur en azote n'est pas fixe : elle varie avec chaque usine et, pour chaque usine, avec le moment et l'époque où le prélèvement a été effectué.

Eaux de lavage des bidons et récipients, elles ne sont que du lait dilué.

Eaux de lavage de la caséine, elles seront un peu plus riches en sérum et en grumeaux de caséine. 
Quelques chiffres, empruntés à DIENERT, montrent les limites entre lesquelles peut varier la composition des eaux de laiterie :

\begin{tabular}{l|c|c|c}
\hline & $\begin{array}{c}\text { Mat. organ. } \\
\text { (mmgr. par litre) }\end{array}$ & $\begin{array}{c}\text { Azote nitrique } \\
\text { (mmgr. par litre) }\end{array}$ & $\begin{array}{c}\text { Matières grasses } \\
\text { (mmgr. par litre) }\end{array}$ \\
\hline Eaux résid. totales .......... & 492 à 2.733 & 58 à 118 & 150 à 290 \\
Eaux de lavage du lait ...... & 331 à 712 & 24 à 51 & 159 à 290 \\
\hline \hline
\end{tabular}

D'autres analyses, citées par DoRNic et CHOLLET, ne sont pas moins suggestives.

\begin{tabular}{|c|c|c|c|c|}
\hline en mmgr. par litre & $\begin{array}{c}\text { Kattein et } \\
\text { Scoofs } \\
\text { Eaux de } \\
\text { lavage de } \\
\text { bidons à } \\
\text { lait }\end{array}$ & Bommer & Calmette & $\begin{array}{c}\text { Daire } \\
\text { Eaux de beur- } \\
\text { reries industri- } \\
\text { elles }\end{array}$ \\
\hline $\begin{array}{l}\text { Degré d'oxydabilité } \quad \text { de } \\
\quad \text { Mno }^{4} \mathrm{~K} \ldots \ldots \ldots \ldots \ldots \ldots \\
\text { Azote organique } \ldots \ldots \ldots \ldots \\
\text { Matières organiques } \ldots \ldots \ldots \\
\text { Matières grasses } \ldots \ldots \ldots \ldots \\
\text { Lactode } \ldots \ldots \ldots \ldots \ldots \ldots \ldots\end{array}$ & $\begin{array}{c}754,7 \\
24,5 \\
300,7 \\
159-290\end{array}$ & $\begin{array}{c}126-4.076,4 \\
7-118 \\
253-2.733 \\
\text { Traces à } \\
731\end{array}$ & \begin{tabular}{c}
\multicolumn{1}{c}{43,6} \\
$1.550-2.135$ \\
$628-1.140$
\end{tabular} & $\begin{array}{c}550-3.050 \\
15-130 \\
215-1.763 \\
80-739 \\
\text { Traces à } \\
0,509\end{array}$ \\
\hline
\end{tabular}

La caséine, la lactalbumine, le lactose (celui-ci en assez faible quantité dans les eaux dé beurreries et de caséineries exemptes de sérum) et les matières grasses sont leurs constituants principaux. Eminemment putrescibles si on les abandonne à elles-mêmes, on voit le lactose se transformer en acide lactique, puis en acide acétique: les albumines passer peu à peu à l'état ammoniacal: les matières grasses subir une lente oxydation.

Le volume des eaux usées, évalué en Charente-Inférieure, au minimum, au triple de la quantité de lait traité quand l'on ne procède qu'à trois lavages de la caséine, s'élève toujours à plusieurs tonnes par jour. Ces quantités sont naturellement sous l'influence de la saison et de la production laitière; moins importantes en hiver, elles augmentent au printemps et en été. Ne sont pas rares les coopératives, qui doivent évacuer quotidiennement 60 à 80 tonnes d'eaux usées.

Lorsqu'on se trouve à proximité d'un cours d'eau (rivière et non simple ruisseau), coulant toute l'année et ayant, même en été, un débit suffisant, le plus simple est évidemment d'y déverser les eaux résiduaires, mais en tenant compte de la température, du débit, de la rapidité du courant, des caractéristiques du lit de la rivière, de la flore et de la faune.

Les eaux de laiterie appauvrissent les rivières en oxygène, nécessaire à l'oxydation des matières organiques. Cet appauvrissement devient incompatible avec la vie des poissons, s'il abaisse la teneur en oxygène à un taux inférieur à 1,5 sur 1.000 .000 . De toute nécessité, la dilution 
devra être considérable pour ne-pas atteindre ce taux limite : elle devra être plus grande encore, lors de l'addition de la moindre quantité de petit-lait, celuici augmente la nocuité des eaux (au taux de $5 \%$ il, détruit radicalement la faune). Si le débit de la rivière est suffisant non seulement, aucune action nocive se fait sentir, mais même les poissons augmentent en nombre et en poids et la végétation aquatique devient plus luxuriante.

Ce déversement dans les eours d'eau, en principe interdit par les règlements administratifs, ne semble pas, dans la pratique, entraîner de gros inconvénients si l'on a soin de tenir compte des réserves précédentes. Il est particulièrement en honneur en Amérique, le long des rivières importantes. La proximité d'un cours d'eau étant rarement réalisée, il faut envisager d'autres procédés.

De nombreuses méthodes ont été préconisées :

Méthodes physiques;

" chimiques;
" biologiques;
" épandage.

que nous allons succintement passer en revue.

\section{Procédés PHYSTques.}

Ce sont la simple décantation, la centrifugation et la filtration.

La décantation est longue. 48 heures au moins sont nécessaires pour que les éléments en suspension se déposent; les substances solubles participent mal à cette opération qui nécessite des réservoirs volumineux, siège de fermentations multiples, surtout en été. Les matières grasses, remontant à la surface, forment une couche isolante qui arrête en partie les mauvaises odeurs : mais celles-ci deviennent infectes, dès que la couche graisseuse est détruite et surtout lors de la vidange des boues. La décantation d'eaux, riches en petit-lait, transforme seulement le laetose en acide lactique sans diminuer le coefficient de putrescibilité.

La centrifugation, trop onéreuse, n'a qu'une action très faible sur les colloïdes : on ne l'a adoptée nulle part.

La filtration se fait par l'intermédiaire de divers systèmes de filtres continus : notamment type JEWELL et FULNER, filtres à sable fin, ̀̀ briques, à lattes de bois : au moyen de matières ealcaires comme des écailles d'huitres pulvérisées, de matières poreuses, comme du mâchefer ou du coke ou encore au moyen de fragments d'ardoise. Un filtre à sable peut filtrer 60 litres par $\mathrm{m}^{2}$, tous les deux jours. Un inconvénient apparait de suite, c'est que la surface filtrante doit être considérable : filtre de 30 mètres de côté pour la plus petite usine. Les matières organiques retenues et la matière grasse (si l'on n'a pas pris la précaution de la retenir au préalable lorsqu'elle remonte à la surface) colmatent rapide- 
ment le filtre qui est mis hors d'usage. La filtration n'empêche pas le passage des principes en solution : les résultats y sont plutôt médiocres. Les essais, les plus favorables, n'ont éliminé que $23 \%$ des matières organiques totales. De tous les systèmes, le filtre à sable serait le meilleur. Il retiendrait davantage de matières organiques, arrêterait un peu mieux celles en solution, réduirait davantage le taux bactérien et augmenterait considérablement la teneur en nitrates par une oxydation plus poussée que les filtres à lattes de bois ou à briques. La filtration est assez employée en Amérique où elle précède tout envoi d'eaux usées dans les rivières ou cours d'eau. Elle ne saurait convenir pour les eaux riches en petit-lait.

Certains auteurs avaient entrevu la possibliité de stériliser et d'épurer les eaux de laiteries par l'action de l'ozone et des rayons ultra-violets : ces opérations, déjà diffieiles pour des eaux limpides, seraient vaines pour celles qui nous occupent, opaques et trop riches.

\section{EPURATION BIOLOGIQUe.}

Avant de commencer l'étude des divers procédés biologiques, il est nécessaire de rappeler les principes qu'imposent les hygiénistes aux eaux dites épurées. Une eau épurée devrait satisfaire aux conditions suivantes :

$1^{\circ}$ L'effluent ne doit pas avoir plus de $30 \mathrm{mmgr}$. de matières organiques par litre;

$2^{\circ}$ Après une incubation de 7 jours, à $30^{\circ}$, l'oxydabilité de cette eaú doit être la même qu'avant cette incubation.

$3^{\circ} \mathrm{Ne}$ dégager aucune odeur d'ammoniaque ou de putréfaction;

$4^{\circ}$ Etre incapable d'intoxiquer les poissons ou de nuire aux animaux, s'abreuvant dans les rivières où elles seront déversées.

MM. CaLmette et Rolants définissaient ainsi l'épuration :

"On doit entendre non pas une clarification plus ou moins complète, comme on l'obtient avec certains réactifs chimiques, mais l'élimination aussi eomplète que possible de toutes les matières putrescibles, soit solides, soit en dissolution, susceptibles de nuire à la vie des poissons ou des plantes ou de compromețtre la santé publique ».

Appliquée dès 1895 pour l'épuration des eaux d'égouts, l'épuration biologique, simple répétition par des moyens plus forts, plus puissants et sur des espaces plus restreints de ce qui se passe dans la nature, ne fut employée qu'assez récemment pour les eaux de laiterie.

L'organe essentiel de l'épuration biologique est le lit bactérien qui constitue en définitive un champ d'épandage idéal où la transformation des matières organiques est poussée au maximum (à surfaces égales, 45 fois plus que dans l'épandage sur le sol).

Le travail aérobie du lait bactérien est préparé par le séjour des eaux usées dans une "fosse septique " où, dans l'épaisseur des couches de liquide, les microbes anaérobies désintègrent la matière organique, la 
dissolvent ou la transforment en gaz. Les eaux séjournent au moins 24 à 48 heures dans cette fosse septique, dont la capacité doit être en rapport avec le volume des eaux, produites en deux à trois jours par I'usine. La fosse septique était autrefois soigneusement couverte; depuis que l'on a constaté que les anaérobies travaillaient parfaitement et sans gêne à l'abri de la couche isolante superficielle, elle n'est plus couverte et n'est qu'un véritable réservoir de décantation.

L'eau arrive ensuite sur les lits bactériens qui sont de deux types bien différents :

10 Lit de contact quand le déversement se fait en masses dans de larges bassins étanches, remplis puis vidés à intervalles réguliers. La surface est submergée au moment de l'arrivée des eaux qui filtrent lentement;

$2^{\circ}$ Lits percolateurs. Les liquides arrivent en pluie (ce résultat est obtenu par l'emploi d'appareils répartiteurs. SPRINCKLER pour les faibles pressions : appareil baladeur pour ́les pressions plus fortes : bee pulvérisateur ou réservoir de chasse pour les charges plus lourdes).

Les lits bactériens sont, en réalité, de simples filtres dont les matériaux hébergent, dans leurs portes ou leurs interstices, des microbes aérobies qui oxydent, à leur passage, les matières organiques en voie de désintégration. La constitution du lit bactérien a, maintes fois, évolué. Les matériaux, les plus poreux et les plus rugueux (coke, charbon, scories, etc...), primitivement estimés comme les meilleurs, ont été abandonnés à cause de leur rapide désagrégation qui contribuait au colmatage. Aussi maintenant a-t-on recours plutôt à de simples pierres calcaires, qui ne sont évidemment pas à l'abri d'une oxydation, mais dont les déchets ne nuisent pas à la perméabilité du filtre. Le colmatage, gros grief contre les lits bactériens, résulte à la fois de la rétention de matières en suspension, mais surtout des substances colloïdales en pseudo-solution: il est difficile à combattre.

Des essais préalables doivent toujours porter sur la grosseur des matériaux, l'épaisseur du lit et la rapidité de filtration : toutes conditions indispensables pour obtenir une épuration convenable et une aération maximum. Elles varient d'ailleurs avec la richesse des eaux en matières organiques en suspension et en ammoniac.

Quand l'affluent est elair, les matériaux fins et une filtration rapide sont indiqués. Si l'affluent est riche, des matériaux plus gros et plus épais conviennent mieux.

Les lits bactériens attaquent surtout les matières en suspension. Un mètre cube épure 4 à 500 litres; on doit répartir l'eau à raison de 1 mètre cube par mètre carré.

Quels sont les résultats, obtenus en laiterie par les lits bactériens? Les Américains, très partisans de cette méthode, prétendent abaisser la teneur bactérienne totale de 90 à $95 \%$ et celle de matières organiques totales de 70 à $75 \%$ : résultat contesté par maints auteurs. Comment 
peut-on expliquer les divergences aussi "grandes que celles relatées? Elles peuvent tenir : à ce que la composition des eaux n'est pas identique dans les diverses usines, à ce que peut-être certains principes intangibles: .n'ont pas été strictement observés dans la construction et le fonctionnement de certaines stations.

Il ne faut pas oublier que les lits bactériens n'épurent que par l'intermédiaire de microorganismes, qui ont, en général, une affinité spéciale pour les milieux à réaction alcaline ou neutre : or, les eaux de laiterie contiennent du lactose, qui, en fermentant, acidifie le milieu, gêne tout d'abord, puis rend impossible dès que l'acidité a atteint un certain degré, tout travail microbien. L'oxydation se ralentit, avant de s'arrêter définitivement: les matières organiques ne sont plus nitrifiées. $\mathrm{Au}$ contraire, l'alcalinisation des eaux (par une certaine quantité de chaux par exemple) faciliterait le travail des aérobies.

Souvent aussi, pour le nettoyage des bâtiments et du sol de la laiterie, sont employés des antiseptiques, puissants sous une faible dose comme les hypochlorites, (eau de javel): mélangés par la suite aux eaux résiduaires, ils stériliseront les fosses septiques et les filtres, rendant problématique toute épuration. Le même fait se constate régulièrement dans les villes pour l'épuration des vidanges; les ménagères, en jetant dans les water-closet, certaines eaux riches en savon ou en eau de javel, empêchent le fonctionnement des Septic Tank et autres appareils du même genre.

Pour être efficace, le travail du lit bactérien doit être lent et réparti sur environ 22 à 24 heures : si l'on raccourcit trop ce délai, en augmentant la vitesse et le volume de l'affluent, le contact avec les microbes nitrificateurs deviendra insuffisant : l'oxydation sera faible. Les filtres réclament un "amorçage " qui leur apportera les bactéries nécessaires. Les germes n'acquièrent que progressivement la propriété de céder l'oxygène ; ils ont besoin d'un « entraînement ». Des prélèvements, effectués peu après. la mise en marche d'une station, mal amorcée et mal entraînée, n'indiqueraient qu'une épuration imparfaite.

Le lit bactérien, agissant par oxydation, doit récupérer son oxygène: en un mot, il doit respirer: cette "respiration" ne peut être réalisée que par l'intermittence du débit des eaux et par la circulation d'air entre les diverses couches de matériaux; cette condition est impérative; nous ne pouvons cependant entrer ici dans certains détails de construction qui ont leur importance.

Nous devons poser une règle absolue : comme pour tous les autres procédés d'épuration, chimiques ou physiques, il est indispensable de recueillir toute la matière grasse, avant que les eaux n'arrivent sur les lits bactériens ; cette récolte peut être aisément réalisée au moyen de chicanes, établies le long du canal d'adduction. Cette récolte évite. le colmatage et les odeurs infectes des boues. 
Dans toutes les installations eritiquées, a-t-on appliqué les principes que nous venons d'énumérer? Il nous est difficile de l'assurer puisque ces stations n'existent qu'à l'étranger et que les descriptions données sont généralement incomplètes. La méconnaissance ou l'oubli d'une seule des précautions indiquées suffit à empêcher tout fonctionnement.

On a reproché aussi aux fosses septiques la pullulation des mouches et des moustiques.

Le mélange de petit-lait et de babeurre aux eaux résiduaires rend l'épuration imparfaite: l'effluent serait encore putrescible.

L'épuration biologique ne doit pas être considérée comme strictement automatique : chaque jour, fosse septique et lit bactérien doivent être soigneusement visités et surveillés.

A côté de la fosse septique primitive, ont pris place d'autres procédés biologiques :

$1^{\circ}$ Fosse Imhoff;

$2^{\circ}$ Boues activées.

Le réservoir Imhoff (fosse de décantation à deux compartiments), est dè construction onéreuse : l'effluent non filtré aurait une teneur bactérienne diminuée de 55 à $70 \%$; et filtré, de 80 à $85 \%$. La réduction des matières organiques serait inférieure à celle des fosses septiques: les filtres se colmateraient aussi plus vite; il est indispensable, dans ce procédé, de tenir compte de la température des boues. Peut-être avantageuse pour les énormes masses des eaux d'égouts, en permettant la eaptation et l'utilisation industrielle des gaz, cette méthode n'est pas à conseiller pour les eaux de laiterie. Les mouches ne tardent pas à pulluler et peuvent entraîner la pollution des beurres et du lait. Cette méthode a été abandonnée en Amérique.

Le procédé d'épuration par les "boues activées", inventé par les chimistes anglais ARDERN et LOCKETT de Manchester, consiste dans la forte agitation en présence de l'air d'un mélange d'eaux résiduaires avee un grand volume de "boues activées ".

Le procédé est très imple en pratique. Les eaux résiduaires, débarrassées des grosses impuretés par décantation, traitement chimique ou biologique, se mélangent dans un bassin, régulateur indispensable, avec un tiers $(1 / 3)$ ou un quart $(1 / 4)$ de leur volume de bones activées par une précédente opération. Elles sont brassées pendant 3 ou 4 heures, au moyen d'un courant d'air ou d'un agitateur mécanique, puis laissées au repos. La boue se dépose; les matières organiques ont, pendant le brassage, été oxydées. Le liquide clair est évacué; la diminution des matières organiques atteindrait 70 ̀̀ $80 \%$. Le temps d'épuration varie évidemment avec la nature de l'eau, son degré de pollution, l'aération et l'agitation.

Une boue est dite «activée », quand elle a acquis la propriété d'adsorber les matières organiques plus ou moins transformées et d'oxyder 
l'azote ammonical en azote nitreux ou nitrique. Cette activation de la boue résulterait soit de l'action des microbes soit de celle des protozoaires: l'explication du mécanisme d'action, encore obscure à fait naître maintes théories.

Cette activation de la boue est une propriété assez spéciale, acquise et persistante. La boue activée, habituée à épurer en milieu agité, oxyde aussi bien l'azote organique ou ammonical dans un liquide au repos, alors qu'une boue ordinaire, transportée dans un milieu agité, serait incapable d'exercer ces oxydations successives. Par agitation continue, on arrive à modifier ses propriétés et à la rendre apte à ce travail en milieu agité et aéré.

Dienert indique que l'on peut obtenir des boues activées de la façon suivante : "l'eau d'égout ou de l'eau additionnée de $3 \%$ de lait est mise dans un bassin, dans lequel on insuffle de l'air pendant 24 heures. Après ce temps, on décante le liquide et l'on ajoute de l'eau nouvelle et du lait dilué. En répétant cette opération toutes les 24 heures, on arrive au bout de trois à quatre semaines à récolter une boue qui possède les propriétés suivantes:

" a) De déposer rapidement et d'avoir l'apparence colloïdale et de petites éponges;

( $b)$ De laisser à sa surface un liquide elair;

“c) D'oxyder les matières organiques ;

"d) De nitrifier l'ammoniac. "

D'après le même auteur, un autre procédé de préparation des boues activées " ayant un très grand pouvoir épurant sur les eaux de laiteries, s'obtient en passant par l'intermédiaire d'une poudre de bioxyde de manganèse active, obtenue en agitant ce corps en présence de l'air et d'eau de rivière pendant 2 à 3 semaines. Cette poudre active est ensuite mise en contact avec les solutions de lait à $2 \%$ qu'on agite en présence d'air, pendant 12 heures ».

(à suivre)

\section{BIBLIOGRAPHIE ANALYTIQUE}

\section{LES LIVRES}

Schetn (H.) et Bergeon (P.). - Les buflesses de Delhi. Tirage à part de la Revue Mensuelle des renseignements sur l'Indo-Chine, $\mathrm{n}^{\mathrm{0}} 1$, janvier 1927.

Les bufflesses de L'Inde, de la race dite de Delhi, ont une aptitude laitière exceptionnelle. Dans leur pays d'origine, elles fournissent 18 à 24 litres de lait par jour, pour un poids vif de 520 à $580 \mathrm{~kg}$. Ces rendements sont bien différents de ceux qu'on observe chez nos bufflesses tonkinoises qui ne donneraient guère que 1 litre à 1 litre $1 / 2$ de lait par jour. 